\title{
Decision Support in Patients with mild Alzheimer's Disease
}

Silke M. Mueller ${ }^{a}$, María García Arias ${ }^{b}$, Gema Mejuto Vázquez ${ }^{\mathrm{c}}$, Johannes

Schiebener ${ }^{\mathrm{a}}$, Matthias Brand ${ }^{\mathrm{a}, \mathrm{d}}$, and Elisa Wegmann ${ }^{\mathrm{a} *}$

${ }^{a}$ General Psychology: Cognition and Center for Behavioral Addiction Research

(CeBAR), University of Duisburg-Essen, Duisburg, Germany; ${ }^{b}$ Gabinete de

Orientación Educativa, University Francisco de Vitoria, Madrid, Spain; ${ }^{c} F u n d a c i o ́ n$

Vianorte-Laguna, Hospital Centro de Cuidados Laguna, Madrid, Spain; ${ }^{d}$ Erwin L.

Hahn Institute for Magnetic Resonance Imaging, Essen, Germany

*Corresponding author:

Elisa Wegmann

General Psychology: Cognition

University of Duisburg-Essen

Forsthausweg 2

47057 Duisburg, Germany

Phone: +49-203-379-2251

Email: elisa.wegmann@uni-due.de

Contact details of all other authors:

Silke M. Mueller

Email: silke.m.mueller@uni-due.de

María García Arias

Email: maria.garciaarias@ufv.es

Gema Mejuto Vázquez

Email: g.mejuto@lagunacuida.org

Johannes Schiebener

Email: johannes.schiebener@uni-due.de

Matthias Brand

Email: matthias.brand@uni-due.de 


\section{Decision support in patients with mild Alzheimer's disease}

Introduction: Making advantageous decisions is a key competence of individuals of all ages. However, previous studies reported a reduction of this competence in patients with neurodegenerative diseases such as Alzheimer's disease, which is explained by impairments of executive functions such as cognitive flexibility or working memory. While previous findings from healthy participants with reduced executive functions showed that support can improve decision making under risk, the study at hand aimed to investigate this effect in patients with mild Alzheimer's disease (mAD).

Method: A group of elderly individuals diagnosed with $\operatorname{mAD}(n=14$; mean MMSE $=24.14, S D=3.18)$ and a control group of healthy age-matched controls $(n=14$; mean MMSE $=29.29, S D=1.98)$ performed a decision-making task (GDT) three times $\left(t_{0}, t_{1}, t_{2}\right)$ with intervals of five to nine days between each: The standard GDT plus other neurocognitive tasks $\left(\mathrm{t}_{0}\right)$, the GDT with supportive information following each decision $\left(\mathrm{t}_{1}\right)$, and again the GDT without decision support $\left(\mathrm{t}_{2}\right)$.

Results: At any time, mAD patients made more disadvantageous decisions than controls. However, the decision-making performance of mAD patients improved significantly with decision support. Interestingly, when the standard GDT was played again $\left(\mathrm{t}_{2}\right), \mathrm{mAD}$ patients' performance remained similar to the performance in the GDT with decision support $\left(t_{1}\right)$. GDT performance correlated consistently with executive function measures in the control group, but only at $\mathrm{t}_{0}$ in the $\mathrm{mAD}$ group.

Conclusions: The findings indicate that supportive information about the riskiness of options can compensate for $\mathrm{mAD}$-related deficits in decision making under risk. Thus, decision support can improve the quality of mAD patients' decisions. Further, it may prevent mAD patients from making highly risky decisions in similar situations in the future. The persistence of decision support should be further investigated as it has relevant implications for every-day decisions that include risks.

Keywords: decision making; Alzheimer's disease; decision support; Game of Dice Task; executive functions 


\section{Introduction}

Decision making determines people's everyday lives from childhood through to old age. Even in old age, people are faced with choices that, in some cases, can have farreaching consequences. For example, decisions can concern medical treatment, contractual agreement, or wealth management. In older age, the risk of developing a neurodegenerative disease increases. However, people are still confronted with such complex decision situations. Neurodegenerative diseases, including Alzheimer's disease (AD), were shown to have negative impact on decision making (for a review see Gleichgerrcht, Ibáñez, Roca, Torralva, \& Manes, 2010). This is not only the case in decisions under ambiguity, which require learning from feedback, but also in decision situations that provide explicit information about possible outcomes, chances, and risks (Sinz, Zamarian, Benke, Wenning, \& Delazer, 2008). These kinds of decisions, i.e. when a priori information about consequences and their probabilities is given, are referred to as decisions under objective risk conditions (for a recent review see Schiebener \& Brand, 2015a). In decision making under objective risk, cognitive functions associated with reflective information processing, such as working memory, executive functions, and ratio processing, especially contribute to advantageous decision-making behaviour (Brand, Schiebener, Pertl, \& Delazer, 2014; Pertl, Zamarian, \& Delazer, 2017; Schiebener et al., 2014; Schiebener, Zamarian, Delazer, \& Brand, 2011). Executive functions comprise different skills that are relevant for cognitive control processes, such as retrieving and integrating information from memory, planning, behavioral inhibition and monitoring, evaluating and categorizing options, or forming and applying a decision-making strategy (Anderson \& Tranel, 2002; Schiebener \& Brand, 2015b). In AD, brain areas associated with executive functions and memory are affected from an early to mid-stage (Braak \& Braak, 1991; P. T. 
Nelson et al., 2012). These impairments are assumed to be the main reason why patients with $\mathrm{AD}$ or other neurodegenerative diseases show deficits in decision-making tasks (Gleichgerrcht et al., 2010). Decision making, however, is not only determined by internal factors, such as cognitive abilities, but also by external and situational factors as suggested by theoretical models (e.g. Finucane \& Lees, 2005; Schiebener \& Brand, 2015a). Some situational factors were found to help individuals with reduced executive functions to make more advantageous decisions in risky situations. For example, the decision situation itself can offer supportive information such as feedback, advice, or warning, which can improve decision making (Brand, Laier, Pawlikowski, \& Markowitsch, 2009; Schiebener, Wegmann, Pawlikowski, \& Brand, 2013; Yaniv, 2004). The current study aims to investigate whether decision support can compensate for decision-making deficits in patients with mild Alzheimer's disease (mAD) in a sustainable manner.

\section{Decision making under risk in neurocognitive diseases}

Patients suffering from neurodegenerative diseases such as AD show significant decision-making impairments in laboratory decision-making tasks (de Siqueira, Yokomizo, Jacob-Filho, Yassuda, \& Aprahamian, 2017; Gleichgerrcht et al., 2010). Most of the studies summarized in a recent review by de Siqueira et al. (2017) investigated patients in early stages of probable mAD and mild cognitive impairment showing decision-making deficits in situations under both ambiguity and risk. The Game of Dice Task (GDT; Brand et al., 2005) is one of the most frequently used tasks to measure decision-making under (objective) risk conditions. In the GDT, a virtual die is thrown and participants are asked to bet on which number will be thrown next. Participants can bet either on a single number or on different combinations of multiple numbers (two, three, or four numbers) simultaneously. Betting on less numbers is 
associated with higher bet amounts, but also with a higher risk of losing. Accordingly, decisions for one number or a combination of two numbers are of high risk and objectively disadvantageous, i.e., the risk of losing is higher than the chance to win. Patients with frontotemporal dementia, dorsolateral prefrontal cortex damage (Bechara \& Martin, 2004; Brand, Kalbe, et al., 2004), Korsakoff's syndrome (Brand et al., 2005), Parkinson's disease (Brand, Labudda, et al., 2004; Euteneuer et al., 2009), and mAD (Delazer, Sinz, Zamarian, \& Benke, 2007) were shown to make significantly more of the highly risky decisions compared to healthy participants. Superior performance in the GDT is associated with high executive functions and other cognitive processes that are involved in the development and application of advantageous strategies (Brand et al., 2014; Delazer et al., 2007; Schiebener \& Brand, 2015b; Schiebener et al., 2014).

Conversely, disadvantageous and highly risky choice behaviour may result from deficits in respective cognitive domains. The study by Delazer and colleagues (2007) supports this view. The analysis of response patterns in the GDT showed that mAD patients (in contrast to healthy controls) did not learn from feedback and did not adapt their strategy despite negative outcomes. The authors assume such disadvantageous decision-making behaviour to result from deficits in learning and executive functioning (Delazer et al., 2007). AD initially affects medial temporal brain regions including the hippocampus, which is important for episodic memory and the construction of mental images (Bird \& Burgess, 2008) and, at later stages, also frontal brain regions appear to be affected. These neurological changes lead AD patients to suffer from executive dysfunctions (Baudic et al., 2006; Binetti et al., 1996; Lafleche \& Albert, 1995) and deficits in especially episodic memory (Bäckman, Small, \& Fratiglioni, 2001; Bondi et al., 2008; Grady et al., 1988; Hodges \& Patterson, 1995; R. G. Morris \& Kopelman, 1986; Perry, Watson, \& Hodges, 2000; Welsh, Butters, Hughes, Mohs, \& Heyman, 1992), even in 
the early stages of the disease. Changes in executive functions and other cognitive skills such as cognitive flexibility, inhibition, and working memory appear to account not only for disease-related but also for general age-related reductions in the decisionmaking performance under objective risk conditions (Brand \& Schiebener, 2013; Liebherr, Schiebener, Averbeck, \& Brand, 2017; Schiebener \& Brand, 2017).

\section{Theoretical classification of decision support}

Theoretical models of decision making suggest internal as well as external factors to influence how decisions are made. The Person-Task-Fit Framework (Finucane \& Lees, 2005; Finucane, Mertz, Slovic, \& Schmidt, 2005) defines that the individual decisionmaking competence is influenced by characteristics of the person (internal) as well as by characteristics of the task and other situational factors (external). This differentiation between internal and external factors has also been applied to the process of decision making under objective risk conditions (Schiebener \& Brand, 2015a). While internal factors comprise individual characteristics of the decision maker (e.g. age, impulsivity, or cognitive abilities), external factors include characteristics of the decision-making situation, e.g. additionally provided information and situational induced states, such as parallel cognitive load or stress. Executive functions and probability processing skills are internal factors, which are of particular importance for making superior decisions under objective risk (Brand et al., 2014; Schiebener et al., 2014). Executive functions (e.g. inhibition, set-shifting, cognitive flexibility) and working memory are assumed to be important for exercising cognitive control (including functions such as reflecting on options, feedback monitoring, planning, and strategy application) and for the evaluation of risks, which is why they are attributed to reflective information processing. Results of experimental studies indicate that executive functions are especially relevant in decision situations where no direct feedback about (long-term) consequences is available (Brand 
et al., 2009; Mueller, Schiebener, Stöckigt, \& Brand, 2017), misleading biases are present (Schiebener, Wegmann, Pawlikowski, \& Brand, 2012), or no support is offered (Schiebener et al., 2013). Accordingly, external influences of the decision situation, such as feedback or supportive information, can have positive effects on decision making under objective risk (see also Figner, Mackinlay, Wilkening, \& Weber, 2009).

Decision support was shown to be capable of improving the quality of decision outcomes and/or of reducing cognitive effort (Engelmann, Capra, Noussair, \& Berns, 2009; Todd \& Benbasat, 1994). In an fMRI study, Engelmann et al. (2009) asked healthy participants to decide multiple times between a fixed amount (safe option) and a lottery (risky option). One group received decision support in terms of an expert's advice on what option to accept and what option to reject. The advice was presented textually above the options. The behavioural results showed that decision making was significantly influenced by the advice. The brain imaging results showed that brain areas associated with calculation and evaluation processes (i.e. the intraparietal sulcus, anterior cingulate cortex, and dorsolateral prefrontal cortex) were less activated in participants who had received support compared to those who had not received support. Consistently, Schiebener and colleagues (2013) found that in decision making under objective risk, as measured by performance in the GDT, decision support in terms of explicit advice had a positive effect on the decision-making performance in healthy subjects. Especially individuals with below-average working memory and executive functions could profit from the explicit advice. In a pre-study, it was tested what kind of advice was most effective for supporting decision making in the GDT. The different types of support were: extended instructions, presentation of exact probabilities, presentation of the maximum gain/loss, additional test trials, and explicit advice on which options are advantageous. The explicit advice was shown to be most effective. 
The authors assumed that advice leads to a reduction of load on prefrontal cortex functions, which are involved in risky decision making (Schiebener et al., 2013). This is consistent with the findings by Engelmann et al. (2009), indicating that advice reduces the activation of brain regions associated with the evaluation of risk information, e.g. the dorsolateral prefrontal cortex and the anterior cingulate cortex. A recent study by Burgio and colleagues (2018) provides evidence that training on executive functions and number processing can improve the decision-making performance under objective risk conditions in patients with mild cognitive impairments. The findings further support the view that deficits in executive functions in neurological patients with mild cognitive impairments are associated with more disadvantageous choice behavior, but that specific support addressing these deficits can help to improve decision making.

\section{Research hypotheses}

The current investigation is based on the study by Schiebener et al. (2013), which showed positive effects of decision support in the GDT for healthy individuals with comparatively reduced working memory and executive functions. Accordingly, we hypothesized that decision support can help mAD patients to make better (/less risky) decisions, as it may reduce the involvement of executive functions and thus compensate for respective disease-related cognitive impairments. Further, it can be assumed that decision support in terms of information about the riskiness of options provides factual knowledge that may be remembered when being confronted with similar decision situations again. Accordingly, we wanted to test the hypothesis that the positive effect of decision support may persist in similar decision-making situations in the future. 


\section{Method}

\section{Participants}

The current study investigated a total of 28 elderly participants, who were recruited and examined at the Alzheimer day center of the Vianorte-Laguna Foundation in Madrid (Spain). All participants performed the center's standard diagnostic procedures. The mAD group consisted of 14 participants ( 4 females, 10 males) with probable mAD diagnosed by the local neurologist according to NINCDS-ADRDA criteria (McKhann et al., 1984). The mean age was $M=77.57(S D=5.96)$ years. The control group consisted of 14 elderly participants (mainly relatives and friends) not meeting the mAD criteria ( 8 females, 6 males), who were of matching age $(M=74.07, S D=7.56), t(26)=$ $-1.36, p=.185$, and gender, $\chi^{2}(1, \mathrm{~N}=28)=2.33, p=.127$. The presence of significant vascular ischemic disorder was excluded according to Hachinski’s ischemia scale (Hachinski et al., 1975). All participants had normal or corrected-to-normal sight and sufficient hearing abilities. The years of school education (mAD: $M=10.14, S D=6.24$; control: $M=13.64, S D=6.78)$ did not differ significantly between groups, $t(26)=1.42$, $p=.167$. Participation was voluntary without payment. The study was approved by the ethics committee of the Hospital Clínico San Carlos in Madrid (Spain). All participants gave written informed consent before participation and were standardly debriefed afterwards. Participants agreed with the inclusion of material pertaining to themselves with the stored data being anonymized.

\section{Design and Instruments}

The current study followed a mixed design. Two groups of participants (betweensubjects factor), namely the mAD patients and the elderly controls, were tested at three different times (within-subjects factor) with intervals of five to nine days (due to 
practical reasons) between each (see Figure 1 for an illustration of the study design). In the first session ( $\mathrm{t}_{0}$ ), all participants performed a decision-making task (the GDT) followed by different neuropsychological tasks assessing cognitive functions. In the second session $\left(t_{1}\right)$, five to nine days from $t_{0}$, both groups performed the GDT again, but with decision support. Support was offered after each decision in terms of information about the riskiness of the respective choice, with the concurrent question whether the participants want to maintain or change their decision. In the third session $\left(\mathrm{t}_{2}\right)$, five to nine days from $\mathrm{t}_{1}$, participants conducted the GDT again in the standard version without decision support.

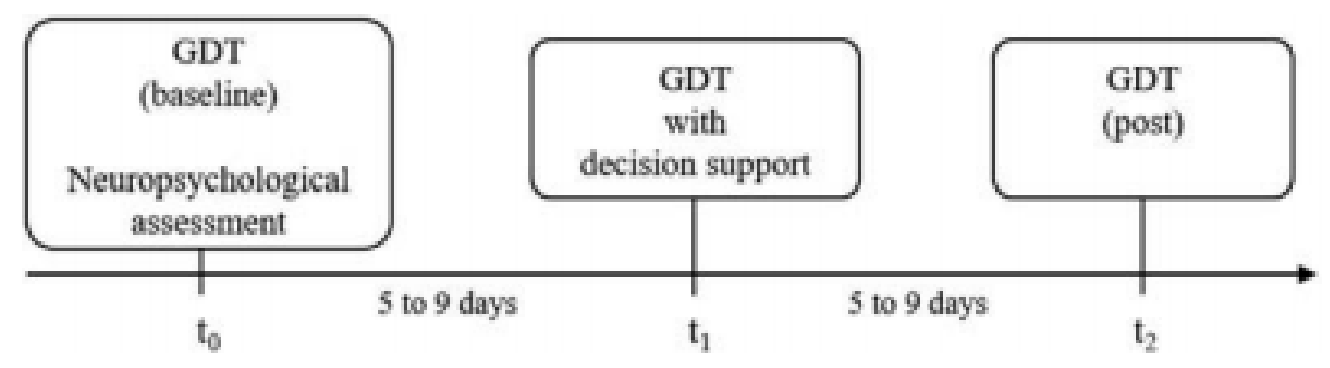

Figure 1. Illustration of the study design with three measurement sessions in each of which the Game of Dice Task was performed.

The three different measurements were completely independent from each other, meaning that in each session the GDT started from the very beginning without the participants having been informed about their performance in previous sessions. Each of the participants was tested individually under similar controlled laboratory conditions. The instructions for each of the tests were given in a standardized manner. All tests were in the local language (Spanish). 


\section{Game of Dice Task (GDT) - standard}

The GDT (Brand et al., 2005) measures decision making under objective risk conditions. The aim is to maximize a virtual capital of $\$ 1,000$ by repeatedly betting on the results of a die roll. Figure 2 illustrates the GDT with its typical decision situation. Bets can be placed either on a single number (1 to 6 ) or on a combination of two (e.g. “3, 4”), three (e.g. “4, 5, 6”), or four numbers (e.g. “1, 2, 3, 4”). Different amounts of numbers are associated with different amounts of virtual money that can either be won or lost. The respective amount is added to the current capital in case the die shows one of the numbers that the bet was placed on, otherwise the amount is subtracted from the current capital. A bet on one single number results in a gain/loss of $\$ 1,000$. Accordingly, due to a winning probability of $1 / 6$ and a risk of losing of $5 / 6$, betting on one single number is the riskiest option. Bets on two numbers result in a gain/loss of $\$ 500$. They are also of high risk, because the chance to win is lower than the probability to lose (2/6 vs. 4/6). Bets on combinations of three or four numbers can result in a gain/loss of $\$ 200$ or $\$ 100$ respectively. Both options are of low risk, and thus termed advantageous, because of a winning probability equal or higher than $50 \%$. The GDT consists of 18 rounds, in each of which a virtual die is thrown, while the betting conditions remain stable. After each decision, participants get explicit feedback about which number had been rolled as well as about the associated gain/loss and the respectively updated current capital. The overall number of advantageous over disadvantageous decisions (GDT net score) serves as the main performance measure. Furthermore, the choices for different combinations of numbers are stored in additional sum scores, with the number of choices for one single number (GDT one number) representing the frequency of decisions with the highest risk. In the current study, participants performed the standard GDT (as described above) in the sessions at $\mathrm{t}_{0}$ and 
$t_{2}$. In the second session $\left(t_{1}\right)$, the participants performed a modified version of the GDT that provides decision support as described below.

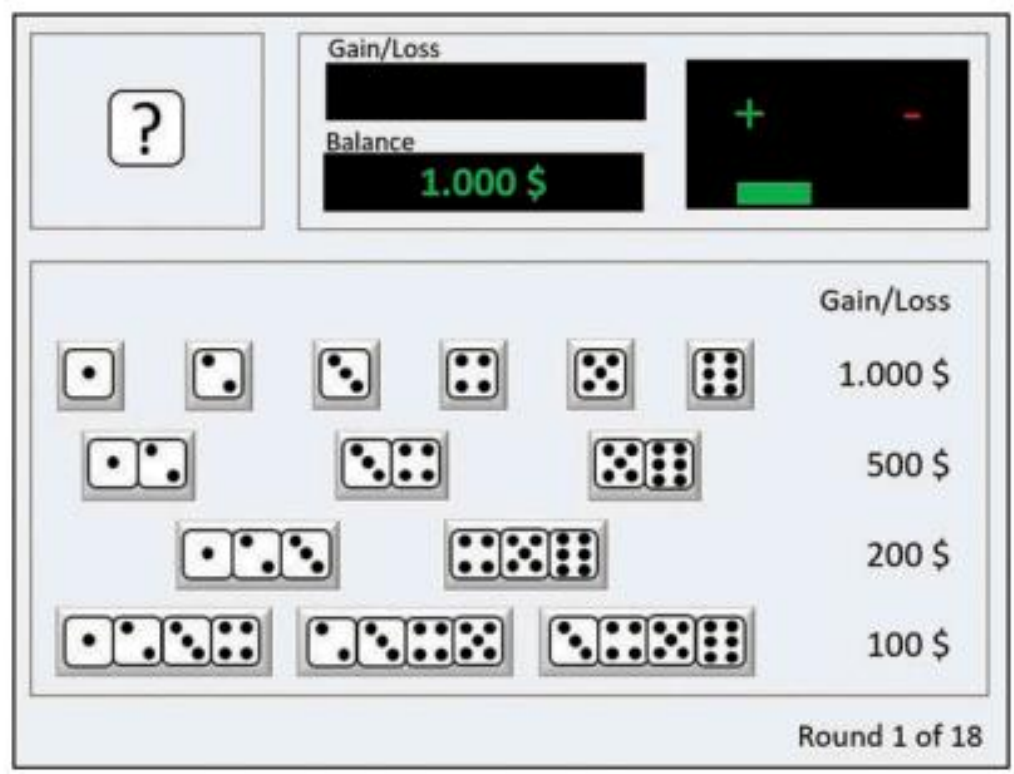

Figure 2. Illustration of a decision trial in the Game of Dice Task (Brand et al., 2005).

\section{Game of Dice Task (GDT) with decision support}

The GDT with decision support follows the same procedure as the standard GDT (see above), except that each decision is followed by a pop-up window that provides information about the riskiness of the just made decision. Concurrently, the participants have the possibility to adjust their decision. For example, when a participant chooses to bet on the combination " 5,6 " (i.e., two numbers), the following information would occur:

You just chose to bet on the following numbers: 56

This decision involves a risk of losing money that is higher than the chance to win money. Do you want to stay with your decision or do you want to make a new decision?

When a participant chooses to bet on a combination of three or four numbers, the supportive information is adjusted accordingly by stating that the decision offers an 
equal/ higher probability to win than to lose. The participant can then either "stay with the decision" or "make a new decision". An exemplary trial of the GDT with decision support is illustrated in Figure 3.

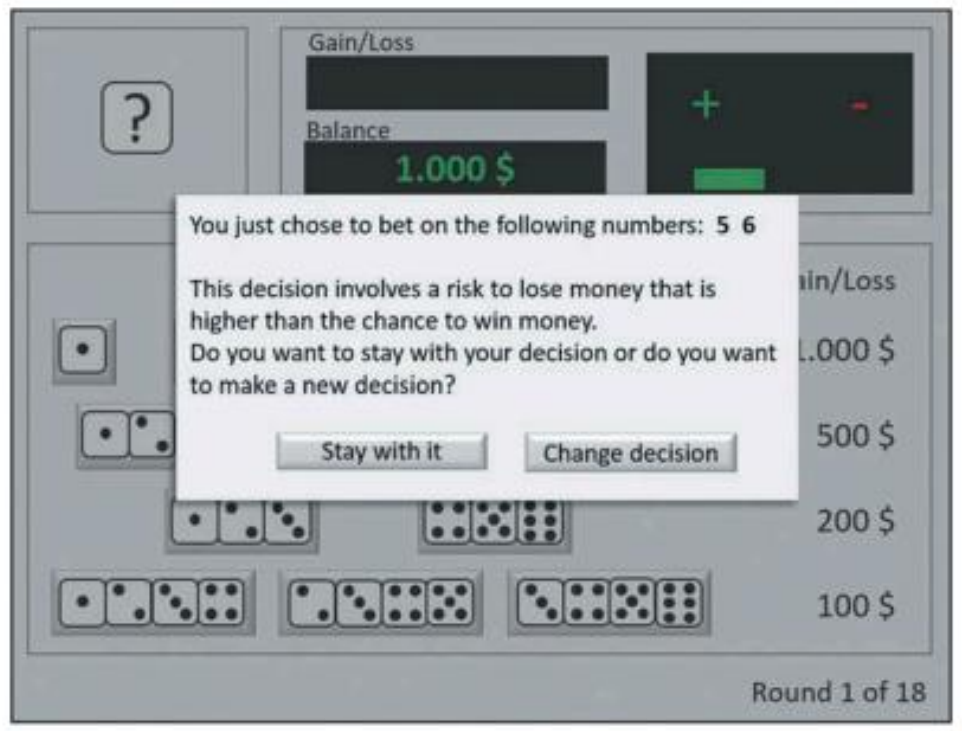

Figure 3. Example of the supportive information following a decision in the Game of Dice Task with decision support.

As in the standard version, the GDT net score (i.e., the number of advantageous minus disadvantageous decisions) and the sum of choices for one number (i.e., the number of decisions for the riskiest options) are calculated as measures of the decisionmaking performance.

\section{Neuropsychological assessment}

As screening instruments for cognitive impairments, we conducted the Spanish version of the Mini-Mental-State Examination (MMSE; Folstein, Folstein, \& McHugh, 1975) by Blesa and colleagues (2001) as well as the Montreal Cognitive Assessment (MoCA; Nasreddine et al., 2005). In the MMSE, scores below 24 indicate (mild) cognitive 
deficits (Tombaugh \& McIntyre, 1992). The MoCA comprises tests for different cognitive domains (attention and focus, executive functions, memory, language, visuoconstructive skills, conceptual thinking, calculation, and orientation) with scores below 17 indicating the presence of mAD symptoms (Freitas, Simões, Alves, \& Santana, 2013).

Executive functioning was measured using a computerized version of the Modified Card Sorting Test (MCST; H. E. Nelson, 1976), which has been used in the previous study by Schiebener et al. (2013). The MCST especially places demands on feedback processing, categorization, and set-shifting. In the MCST, a specific card should be sorted to one of four decks, each of which contain different kinds of symbols. Cards can be sorted after colour, shape, or quantity of the symbols. Once sorted correctly (indicated by a happy smiley face in the computer version), the next card must be sorted according to the same rule. The rule has to be followed until it is told that the sorting-rule has changed. The total number of errors (i.e. the sum of perseverative and non-perseverative errors) was calculated. Higher scores indicate weaker executive functions.

Short-term and working memory were assessed using the Digit Span test (forward and backward) of Wechsler's Adult Intelligence Scale (WAIS; Wechsler, Coalson, \& Raiford, 1997) with the "forward" subtest representing verbal attention span and the "backward" subtest representing verbal working memory. Furthermore, verbal fluency tests (regarding words starting with "M" and animals) were conducted (Benton, Hamsher, \& Sivan, 1994; Spreen \& Strauss, 1998). Thereby, the number of correctly mentioned words within a period of one minute was counted.

The Stroop Color-Word Test (SCWT; Stroop, 1935) was administered for measuring inhibition of cognitive interference. The first two "congruent" trials of the 
SCWT require to (1) read colour-words and (2) name colours of coloured boxes. The third "incongruent" trial asks participants to name the colours of colour-words, which are inconsistent (e.g. the word "blue" is printed in green ink with "green" being the correct answer). Thus, in this third trial, participants are required to perform a less automated task (i.e. to name the colour) by inhibiting the interference caused by a more automated task (i.e., reading the word; Ivnik, Malec, Smith, Tangalos, \& Petersen, 1996; MacLeod \& Dunbar, 1988). Correct reactions within a period of 45 seconds served as the performance measure in each of the three trials.

\section{Statistical analyses}

The statistical analyses were carried out with SPSS 24.0 (IBM SPSS Statistics, released 2016). Parametric tests were applied despite the small sample size (see also Skovlund \& Fenstad, 2001) with the GDT net score, which was normally distributed. A mixed ANOVA tested overall effects of group (between), time of measurement (within), and the interaction of both. In case sphericity was violated, $d f$ s were adjusted by Greenhouse-Geisser correction. According to Cohen (1988), partial eta squared of $\eta^{2} p \geq$ .01 indicates a small, $\eta^{2} \geq .06$ indicates a medium, and $\eta^{2} \geq .14$ indicates a large effect. Furthermore, separate analyses for the within and between factors were carried out in order to examine the effects of both group and decision support in more detail. Within-subjects effects were analysed by use of repeated measures ANOVAs carried out separately for each group (with GDT net score as dependent variable). T-tests for dependent samples were used for post-hoc pairwise comparisons. Friedman tests were calculated (with GDT one number as dependent variable) as the non-parametric equivalent of the repeated measures ANOVA. The between-subjects effect was further analysed with post-hoc pairwise comparisons between groups at the different time points of measurement using T-tests for independent samples (GDT net score) and 
Mann-Whitney-U-tests as the non-parametric equivalent (with GDT one number as dependent variable). Pearson product-moment correlations were calculated to test for bivariate correlations between the performance scores of the neuropsychological tests (MCST, Stroop test, verbal fluency task, Digit Span test) and the decision-making performance (GDT net score). Coefficients of $d \geq 0.2$ and $r \geq .1$ indicate small, $d \geq 0.5$ and $r \geq .3$ indicate medium, and $d \geq 0.8$ as well as $r \geq .5$ indicate large effects (Cohen, 1988). Dependent correlation coefficients were compared using the formula by Steiger (1980).

\section{Results}

\section{Neuropsychological assessment}

The mAD group showed lower performance scores than the control group in all of the neuropsychological tasks (see Table 1). The difference in MCST performance was marginally significant, with $\mathrm{mAD}$ patients making a higher number of errors than the elderly controls. The mAD group showed mean MoCA and MMSE scores below the respective cut-offs indicating cognitive deficits. In contrast, the control group showed mean scores above the respective cut-offs.

Table 1. Descriptive statistics and comparison of the neuropsychological measures between mAD and control group.

\begin{tabular}{|c|c|c|c|c|c|c|}
\hline & \multirow{2}{*}{$\frac{\mathrm{mAD}}{M(S D)}$} & \multirow{2}{*}{$\begin{array}{l}\text { Control } \\
M(S D)\end{array}$} & \multicolumn{2}{|c|}{ Comparison } & \multirow[b]{2}{*}{$p$} & \multirow[b]{2}{*}{$d$} \\
\hline & & & $d f$ & $t$ & & \\
\hline MoCA total & $16.64(2.90)$ & $23.50(2.77)$ & 26 & 6.40 & $<.001$ & 2.42 \\
\hline MMSE total & $24.14(3.18)$ & $29.29(1.98)$ & 26 & 5.13 & $<.001$ & 1.94 \\
\hline MCST errors & $18.14(9.04)$ & $12.07(7.22)$ & 26 & -1.96 & .060 & 0.74 \\
\hline MCST categories & $2.00(2.15)$ & $4.00(1.80)$ & 26 & 2.67 & .013 & 1.01 \\
\hline Digit Span (forward) & $5.71(1.86)$ & $9.14(3.08)$ & 26 & 4.00 & .001 & 1.35 \\
\hline Digit Span (backward) & $3.21(1.31)$ & $5.79(1.48)$ & 26 & 5.00 & $<.001$ & 1.84 \\
\hline Digit Span (sum) & $8.93(2.81)$ & $14.86(4.09)$ & 26 & 4.00 & $<.001$ & 1.69 \\
\hline Fluency (words with M) & $7.71(2.73)$ & $13.86(5.72)$ & 18.62 & 3.63 & .002 & 1.37 \\
\hline Fluency (animals) & $9.43(3.99)$ & $17.50(5.73)$ & 26 & 4.00 & $<.001$ & 1.63 \\
\hline Stroop, words (correct) & $72.00(18.69)$ & $89.86(26.80)$ & 26 & 2.00 & .051 & 0.77 \\
\hline Stroop, colors (correct) & $43.14(15.02)$ & $53.07(16.89)$ & 26 & 2.00 & .112 & 0.62 \\
\hline Stroop, interference (correct) & $18.00(8.24)$ & $27.86(11.04)$ & 26 & 3.00 & .013 & 1.01 \\
\hline
\end{tabular}




\section{GDT performance}

The GDT performance had been measured at three times: at $\mathrm{t}_{0}$ (standard GDT), $\mathrm{t}_{1}$ (GDT with decision support), and $\mathrm{t}_{2}$ (standard GDT) in both mAD patients and elderly controls. Figure 4 illustrates the mean GDT performance of the mAD group and the control group in each session (see Table 2 for the descriptive values). Multiple analyses were performed in order to investigate the effect of decision support on GDT performance (a) overall as well as (b) within each group and (c) between the two groups.

\section{Overall effect of decision support}

We analysed overall effects of performing the GDT with and without decision support on the decision-making performance (GDT netscore), taking into account both the within factor "time of measurement" $\left(\mathrm{t}_{0}, \mathrm{t}_{1}, \mathrm{t}_{2}\right)$ and the between factor "group" (mAD and control). The results of the mixed ANOVA showed an overall effect of "time of measurement", $F(2,52)=4.99, p=.010, \eta^{2} p=.161$, as well as of "group", $F(1,26)=$ $7.05, p=.013, \eta^{2} p=.213$. These main effects indicate that, overall, the GDT net scores improved from $t_{0}$ to $t_{2}$ and that $\mathrm{mAD}$ patients, overall, showed lower net scores than controls. The interaction of the two factors had no significant effect, $F(1,26)=0.44, p$ $=.511, \eta_{p}^{2}=.017$.

Although the interaction between "time of measurement" and "group" was not significant, further analyses were performed in order to examine both effects in more detail, and to test the hypothesis whether decision support can help mAD patients to make more advantageous decisions under objective risk conditions. Therefore, we firstly analysed the effect of decision support (in terms of within-subjects differences in GDT performance between the three measurements) separately for mAD patients and 
elderly controls. Secondly, the between-group effect was analysed in more detail by comparing mAD patients with the elderly controls regarding their GDT performance in each session.

\section{Within-subjects comparisons per group}

Within-subjects differences regarding the GDT performance (GDT net score) in the different sessions $\left(t_{0}, t_{1}, t_{2}\right)$ were analysed separately for each group by the use of repeated measures ANOVAs and respective post-hoc pairwise comparisons. Figure 4 illustrates the mean GDT performance scores at $t_{0}, t_{1}$, and $t_{2}$ separately for mAD patients and controls.

Within the mAD group, "time of measurement" had a significant overall effect, $F(2,26)=3.96, p=.032, \eta^{2} p=.234$. Post-hoc pairwise comparisons with dependent ttests showed that $\mathrm{mAD}$ patients had a significantly higher GDT net score at $\mathrm{t}_{1}$ (with support) compared to $t_{0}$ (without support), $t(13)=-2.58, p=.023$. The difference in mAD patients' GDT performance between $\mathrm{t}_{1}$ and $\mathrm{t}_{2}$ was not significant, $t(13)=1.26, p=$ .229 (see Table 2 for the descriptive values). Because of the small sample size, we also concentrated on effect sizes for the interpretation of the results rather than on significance levels only (Aarts, van den Akker, \& Winkens, 2014). Looking at the effect size coefficients, the effect of the performance difference within mAD patients between $\mathrm{t}_{0}$ (without support) and $\mathrm{t}_{1}$ (with support) was almost large, while the difference between $t_{1}$ (with support) and $t_{2}$ (without support) was rather small (see Figure 4 A for the respective values of $d$ ).

Within the control group, "time of measurement" had no effect on GDT net score, $F(2,26)=1.21, p=.314, \eta^{2} p=.085$ (see Table 2 for the descriptive values). 


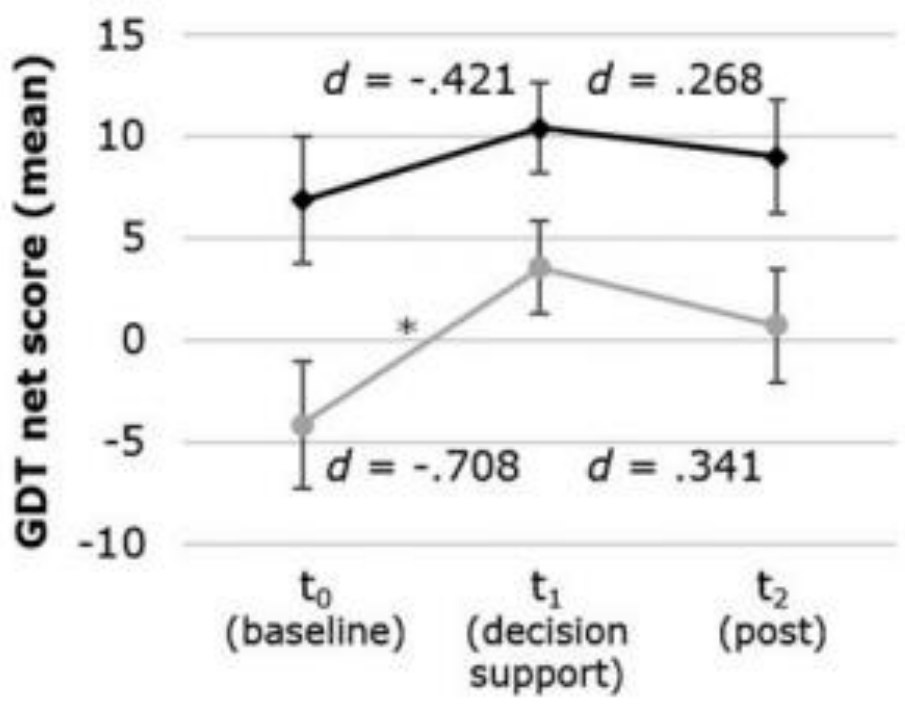

Figure 4. Mean performance in the Game of Dice Task (GDT) at each of the three measurement sessions separated by group (control and mAD) and effect sizes of the session-wise comparisons within each group. Effect size coefficients, $d$ s, corrected for dependence between means according to Morris and DeShon's (2002) equation 8. Error bars represent SEs. ${ }^{*} p<.05$.

Additionally, we had a look at the number of decisions for the highest-risk option (GDT one number) as the dependent variable, because this score was often used in previous GDT studies with clinical samples. Since the variable "GDT one number" was not normally distributed, we performed equivalent non-parametric analyses. In the mAD group, on a descriptive level, the median number of highest-risk choices at baseline decreased after decision support and increased again in the third session (see Table 2 for the descriptive values). However, the results of the Friedman test showed no significant effect of the factor "time of measurement" within the mAD group, $\chi^{2}=2.15, p=.341$. In the control group (see Table 2 for the descriptive values), the Friedman test also revealed no effect of "time of measurement", $\chi^{2}=0.27, p=.875$. 


\section{Between-subjects comparisons per time of measurement}

In order to further investigate the overall effect of "group", we compared the GDT performance between mAD patients and controls session-wise. The results showed that the mAD group had significantly lower GDT net scores compared to the control group at all times (see Table 2).

According to the previous procedure, we additionally compared the number of high-risk decisions (GDT one number) using respective non-parametric tests. The results of the Mann-Whitney-U tests showed that, in each session, mAD patients made significantly more of the high-risk choices compared to the elderly controls (see Table 2).

Table 2. Descriptive statistics and between-group comparisons of the GDT performance at each time of measurement.

\begin{tabular}{|c|c|c|c|c|c|c|c|}
\hline \multirow[b]{2}{*}{ GDT net score } & \multicolumn{2}{|c|}{$\begin{array}{c}\text { mAD } \\
(n=14)\end{array}$} & \multicolumn{2}{|c|}{$\begin{array}{c}\text { Control } \\
(n=14) \\
\end{array}$} & \multicolumn{3}{|c|}{ Comparison } \\
\hline & $M$ & $S D$ & $M$ & $S D$ & $t$ & $p$ & $d$ \\
\hline $\mathrm{t}_{0}$ (baseline) & -4.14 & 12.29 & 6.86 & 11.11 & 2.48 & .020 & 0.93 \\
\hline$t_{1}$ (decision support) & 3.57 & 9.22 & 10.43 & 7.57 & 2.15 & .041 & 0.81 \\
\hline $\mathrm{t}_{2}$ (post) & 0.71 & 10.51 & 9.00 & 10.34 & 2.10 & .045 & 0.80 \\
\hline GDT one number (sum) & $M d n$ & IQR & Mdn & IQR & $U$ & $p$ & $d$ \\
\hline $\mathrm{t}_{0}$ (baseline) & 5.50 & 15 & 0.00 & 2 & 33.00 & .002 & 1.37 \\
\hline$t_{1}$ (decision support) & 2.50 & 6 & 0.00 & 3 & 53.50 & .039 & 0.84 \\
\hline$t_{2}$ (post) & 4.50 & 5 & 0.00 & 2 & 23.50 & $<.001$ & 1.70 \\
\hline
\end{tabular}

Note. $\mathrm{mAD}=$ mild Alzheimer's disease; $\mathrm{GDT}=$ Game of Dice Task; IQR $=$ interquartile range; $\mathrm{t}_{0}, \mathrm{t}_{1}, \mathrm{t}_{2}=$ time points of measurement.

\section{Cognitive correlates of GDT performance with and without support}

In order to test associations between cognitive functions measures (MCST, Stroop test, verbal fluency task, Digit Span test) and GDT performance (GDT net score) within mAD patients and elderly controls, Pearson correlations between the respective variables were calculated separately for both groups. The results showed that, in the mAD group as well as in the control group, the GDT net score at the baseline measurement $\left(\mathrm{t}_{0}\right)$ was negatively correlated with MCST errors (see Table 3 ). This indicates positive associations between executive functions and GDT performance. For 
the $\mathrm{mAD}$ group, this correlation did not remain significant, neither at $\mathrm{t}_{1}$ (with decision support) nor at $\mathrm{t}_{2}$ (without decision support). Comparisons of the dependent correlation coefficients (within mAD patients) showed that the correlation between MCST and GDT performance at $\mathrm{t}_{0}$ differed significantly from that at $\mathrm{t}_{1}, z=-1.86, p=.031$ (onetailed). For the control group, in contrast, the correlation between MCST and GDT performance remained significant at $t_{1}$ and $t_{2}$ with decreasing but still large effect sizes (see Table 3). The other cognitive function measures (Stroop, verbal fluency, Digit Span) did not show consistent correlation patterns with the GDT net score in neither group. The only two exceptions were the correlation with Stroop performance (incongruent trial) in $\mathrm{mAD}$ patients at $\mathrm{t}_{1}$ and the correlation with Digit Span performance (forward) in the control group at $\mathrm{t}_{0}$ (see Table 3 ).

Table 3. Correlations of cognitive abilities with GDT performance at three different time points of measurement separated by group.

\begin{tabular}{|c|c|c|c|c|c|c|}
\hline & \multicolumn{3}{|c|}{ mADGDT net score } & \multicolumn{3}{|c|}{ ControlGDT net score } \\
\hline & $\mathrm{t}_{0}$ & $t_{1}$ & $\mathrm{t}_{2}$ & $\mathrm{t}_{0}$ & $t_{1}$ & $\mathrm{t}_{2}$ \\
\hline MCST errors & $-.741^{* *}$ & -.320 & -.434 & $-.816^{* *}$ & $-.676^{* *}$ & $-.597^{*}$ \\
\hline Stroop, words (correct) & .025 & .102 & 337 & -.191 & -.107 & -.123 \\
\hline Stroop, colors (correct) & .147 & .246 & 368 & -.402 & -.169 & -.170 \\
\hline Stroop, interference (correct) & .395 & $.707^{* *}$ & .416 & .030 & .130 & .050 \\
\hline Fluency names (correct) & .026 & .361 & .136 & .067 & .300 & .364 \\
\hline Fluency animals (correct) & -.281 & .264 & -.099 & -.336 & -.101 & -.053 \\
\hline Digit Span forward & .234 & .343 & 382 & $-.619^{*}$ & -.490 & -.285 \\
\hline Digit Span backward & -.342 & -.068 & .044 & -.110 & .050 & .076 \\
\hline Digit Span sum & -.005 & .194 & .272 & -.515 & -.326 & -.175 \\
\hline
\end{tabular}

\section{Discussion}

The current study investigated the effect of decision support in situations under objective risk conditions in mAD patients and healthy elderly controls. All participants were tested individually in three different measurement sessions (with breaks of five to nine days between each). In each of the sessions, the participants performed the GDT as a measure of decision making under risk: first, the standard GDT $\left(\mathrm{t}_{0}\right)$, then the GDT with decision support $\left(t_{1}\right)$, and then again the standard GDT $\left(t_{2}\right)$. In each session, mAD 
patients made significantly more disadvantageous decisions compared to the elderly controls. However, mAD patients showed a significant performance increase from $t_{0}$ to $t_{1}$ indicating a positive effect of decision support. Within the control group, the GDT performance with and without decision support did not differ significantly.

The results support the hypothesis that decision support can improve the decision-making performance in patients with $\mathrm{mAD}$. This finding is of relevance as deciding among options with objective risks is important for everyday-decisions, but also for health-related situations such as choosing between medications with different risks of side effects or chances of recovery. The decision-making improvement in $\mathrm{mAD}$ patients was indicated by a lower number of very risky choices and a higher ratio of advantageous decisions in the GDT with decision support $\left(\mathrm{t}_{1}\right)$ compared to the GDT without decision support $\left(\mathrm{t}_{0}\right)$. Interestingly, when mAD patients then played the standard GDT without support again $\left(\mathrm{t}_{2}\right)$, they performed comparably to the session with decision support $\left(\mathrm{t}_{1}\right)$. At all times of measurement, mAD patients showed more disadvantageous decision-making behaviour compared to healthy controls. This is in accordance with previous studies on decision making under risk in $\mathrm{AD}$ and other neurodegenerative diseases (Delazer et al., 2007; Gleichgerrcht et al., 2010; Sinz et al., 2008). Moreover, the control group performed equally well in all sessions. Accordingly, the effect of time of measurement was significant only for the mAD group.

The findings indicate that decision support, in terms of explicit information about the riskiness of initially preferred options, can improve decision making under objective risk conditions in $\mathrm{mAD}$ patients. According to previous findings, $\mathrm{mAD}$ patients may have performed poor in the initial standard decision-making task $\left(\mathrm{t}_{0}\right)$ because of deficits to adapt the decision-making behaviour to negative feedback from previous trials (see Delazer et al., 2007). When playing the task again but with decision 
support $\left(\mathrm{t}_{1}\right), \mathrm{mAD}$ patients on average made more advantageous than disadvantageous decisions and chose high-risk options significantly less frequently than before.

The results extend the findings by Schiebener and colleagues (2013). They showed that decision support in terms of explicit information about the riskiness of options had a compensatory effect in healthy adults with reductions in executive functions, such as cognitive flexibility and working memory. The current study indicates that this kind of support can have positive effects on subjects that begin to suffer from $\mathrm{AD}$, which is characterized by executive dysfunctions even in the early stages of the disease (Baudic et al., 2006; Bélanger, Belleville, \& Gauthier, 2010; Lafleche \& Albert, 1995). The lack of adapting behaviour according to the feedback from previous decisions may be a reason for mAD patients' diminished decisionmaking performance under objective risk (Delazer et al., 2007). This lack can be assumed to be due to impairments in cognitive flexibility, working memory, and episodic memory (e.g., Baudic et al., 2006). Confronting decision-makers with information about the riskiness of specific alternatives (as it has been done in the current study), may shift demands from episodic memory (involved in learning from experience) to semantic memory, which seems to be less affected by AD (e.g., Hodges, Salmon, \& Butters, 1990). This is only one speculative explanation, however, it further fits to Bird and Burgess (2008), who state that semantic memory is not likely to dependent on the hippocampus - a region that is critically affected by mAD - as it does not necessarily require the construction of mental images. They assume that also complex long-term representations can be memorized without involving the hippocampus, as long as they are not associated with the construction of a mental picture of the environment. Accordingly, mAD patients might have been able to store the semantic association between options and respective risks, which was given 
explicitly by the supportive information. Further, they might have also been able to retrieve these semantic associations (rather than experiences) several days later. This may explain why mAD patients performed similarly in the second $\left(\mathrm{t}_{1}\right)$ and third sessions $\left(t_{2}\right)$, although the latter was without decision support. Here (at $\left.t_{2}\right), \mathrm{mAD}$ patients might have recalled the information about the riskiness of certain options (given at $t_{1}$ ) from semantic memory, which prevented them from choosing options where the risk of losing is much higher than the chance to win. Accordingly, the results of the current study let assume that decision support in terms of explicit information about the riskiness of options may have a longer-lasting positive effect for $\mathrm{mAD}$ patients in case they are confronted with similar decision situations in the future.

However, we would like to note that this explanation is speculative and that, from the current study, no reasonable conclusions can be drawn on the reasons why mAD patients showed a trend towards an improvement of the decision-making performance after support, which further tended to remain stable over a certain period of time. It is also possible that the supportive information has led mAD patients to reason more about the decision situation and the riskiness of given options. For example, Pertl et al. (2017) showed that decision making was superior in individuals who reasoned about the given options before performing the decision task compared to those who did not. Also, the provided supportive information might have served as a kind of additional feedback, which can have positive effects on upcoming decisions (Brand, 2008; Lejarraga \& Gonzalez, 2011; Mueller et al., 2017). We also acknowledge that practice effects in terms of a growing familiarity with the task and the presented options could be responsible for the persistence in $\mathrm{mAD}$ patients' task performance. The possibility of practice effects being a confounding factor in repeated measures should 
also be considered in studies with patients suffering from $\mathrm{mAD}$ or other neurodegenerative diseases (Goldberg, Harvey, Wesnes, Snyder, \& Schneider, 2015).

In the control group, the decision-making performance did not significantly improve with decision support, which is in contrast to the results reported by Schiebener et al. (2013). However, it has to be mentioned that the current sample differed substantially from the sample in the before mentioned study, both in respect of size and mean age. The group of elderly controls, had already performed well at the first measurement and remained at a comparably high level at all measurements. That was to be expected as previous research indicates that decision making under risk is less affected by normal aging than decisions under ambiguity (Zamarian, Sinz, Bonatti, Gamboz, \& Delazer, 2008). Elderly controls’ decision-making performance was consistently associated with executive functions, which is in accordance with studies on aging and decision making (for a review see Liebherr et al., 2017), whereby the mean decision-making performance was highest with decision support $\left(\mathrm{t}_{1}\right)$. In contrast, mAD patients seemed to benefit more from decision support. Their decision-making performance, which was initially very poor, significantly increased with decision support.

In the present study, the initial GDT performance (at $t_{0}$ ) was strongly associated with cognitive flexibility and set-shifting abilities in both mAD patients and the elderly controls. Correlations of the decision-making performance with individual differences in other cognitive domains, such as verbal working memory or inhibition of cognitive interference occurred only occasionally. This adds to the inconsistent findings on associations between executive functions and decision making in $\mathrm{mAD}$ patients (Delazer et al., 2007; Sinz et al., 2008) and also in healthy participants (Brand et al., 2014; Schiebener \& Brand, 2015b; Schiebener et al., 2014). The current sample of 
mAD patients was too small to test for possible moderating effects of executive functions on the decision-making performance with and without feedback. Whether patients with less affected (compared to those with more affected) executive functioning would profit more or less from decision support should be subject to future investigations. A closer look at patients' individual differences in specific executive functions may provide valuable insights into what kind of support is most effective for each individual.

The current findings have practical implications for supporting patients in risky decision-making situations. According to the current results, providing explicit information about the riskiness of specific decision options may prevent mAD patients (and perhaps even patients with other neurological diseases) from making highly risky choices. Especially in the earlier stages of the disease, patients could be supported with easily understandable information, which may improve decision making especially in situations in their daily lives that involve choosing between options of different risks. For example, care staff and relatives could inform mAD patients more explicitly, in a clear and easily understandable manner, about the risks of specific actions, thus supporting decisions regarding, for example, healthy daily nutrition, correct use of medication, or even protection from fraudsters. The current study gives a first hint on that information about risky decision situations could be a way of compensating for cognitive impairments that are present in mAD. However, even if future studies will prove this kind of support to be effective, it will probably lose its usefulness as the disease progresses. It is possible that a combination of different supportive interventions is needed to improve decision making in AD patients. Recently, Burgio et al. (2018) showed that training patients with mild cognitive impairments in executive functions and numerical processing can help to compensate for deficits in decision making under 
objective risk. Future studies should investigate whether and which actions, such as cognitive training and explicit advice, are complementary, making it possible to prevent patients with cognitive impairments from making highly risky decisions.

As a limitation, we cannot exclude practice effects due to task repetition in the current study design. Further, the variability in the time intervals between the measurements could have led to differential influences of learning and memory. However, it is unlikely that the decision-making improvement solely resulted from learning because of two reasons: On the one hand, a period of about one week was between each investigation, while mAD patients are assumed to be already impaired in integrating information from feedback occurring just a few seconds before the same decision situation appears again (Delazer et al., 2007). On the other hand, if learning effects had led to increased decision-making performance, one would have expected participants to perform best within the last investigation. However, performance measures for both groups tended to decrease rather than increase from $t_{1}$ to $t_{2}$. Future studies may compare the decision-making performance with and without decision support within two groups of mAD patients. Future studies should also ask the patients about their knowledge about the riskiness of the given options, both after having performed the task with and without decision support. Furthermore, research on positive effects of decision support could be extended to patients with other neurodegenerative diseases that include executive dysfunctions.

\section{Disclosure of Interest}

The authors report no conflict of interest. 


\section{Figures}

Figure 5. Illustration of the study design with three measurement sessions in each of which the Game of Dice Task was performed.

Figure 6. Illustration of a decision trial in the Game of Dice Task (Brand et al., 2005).

Figure 7. Example of the supportive information following a decision in the Game of Dice Task with decision support.

Figure 8. Mean performance in the Game of Dice Task (GDT) at each of the three measurement sessions separated by group (control and mAD) and effect sizes of the session-wise comparisons within each group. Effect size coefficients, $d$ s, corrected for dependence between means according to Morris and DeShon's (2002) equation 8. Error bars represent SEs. $* p<.05$. 


\section{References}

Aarts, S., van den Akker, M., \& Winkens, B. (2014). The importance of effect sizes. The European journal of general practice, 20(1), 61-64. doi:10.3109/13814788.2013.818655

Anderson, S. W., \& Tranel, D. (2002). Neuropsychological consequences of dysfunction in human dorsolateral prefrontal cortex. In F. Boller \& J. Grafman (Eds.), Handbook of neuropsychology (Vol. 7, pp. 145-156). Amsterdam: Elsevier.

Bäckman, L., Small, B. J., \& Fratiglioni, L. (2001). Stability of the preclinical episodic memory deficit in Alzheimer's disease. Brain, 124(1), 96-102. doi:10.1093/brain/124.1.96

Baudic, S., Dalla Barba, G., Thibaudet, M. C., Smagghe, A., Remy, P., \& Traykov, L. (2006). Executive function deficits in early Alzheimer's disease and their relations with episodic memory. Archives of Clinical Neuropsychology, 21(1), 15-21. doi:10.1016/j.acn.2005.07.002

Bechara, A., \& Martin, E. M. (2004). Impaired decision making related to working memory deficits in individuals with substance addictions. Neuropsychology, 18(1), 152-162. doi:10.1037/0894-4105.18.1.152

Bélanger, S., Belleville, S., \& Gauthier, S. (2010). Inhibition impairments in Alzheimer's disease, mild cognitive impairment and healthy aging: Effect of congruency proportion in a Stroop task. Neuropsychologia, 48(2), 581-590. doi:10.1016/j.neuropsychologia.2009.10.021

Benton, A. L., Hamsher, K., \& Sivan, A. B. (1994). Multilingual Aphasia Examination: Manual of instructions. Iowa City: AJA Associates.

Binetti, G., Magni, E., Padovani, A., Cappa, S., Bianchetti, A., \& Trabucchi, M. (1996). Executive dysfunction in early Alzheimer's disease. Journal of Neurology, Neurosurgery \& Psychiatry, 60(1), 91-93.

Bird, C. M., \& Burgess, N. (2008). The hippocampus and memory: insights from spatial processing. Nature Reviews Neuroscience, 9(3), 182-194. doi:10.1038/nrn2335

Blesa, R., Pujol, M., Aguilar, M., Santacruz, P., Bertran-Serra, I., Hernández, G., . . . Group, N. (2001). Clinical validity of the 'mini-mental state'for Spanish speaking communities. Neuropsychologia, 39(11), 1150-1157. doi:10.1016/S0028-3932(01)00055-0

Bondi, M. W., Jak, A. J., Delano-Wood, L., Jacobson, M. W., Delis, D. C., \& Salmon, D. P. (2008). Neuropsychological contributions to the early identification of Alzheimer's disease. Neuropsychology review, 18(1), 73-90. doi:10.1007/s11065-008-9054-1

Braak, H., \& Braak, E. (1991). Neuropathological stageing of Alzheimer-related changes. Acta neuropathologica, 82(4), 239-259. doi:10.1007/BF00308809

Brand, M. (2008). Does the feedback from previous trials influence current decisions? A study on the role of feedback processing in making decisions under explicit risk conditions. Journal of neuropsychology, 2(2), 431-443. doi:10.1348/174866407X220607

Brand, M., Fujiwara, E., Borsutzky, S., Kalbe, E., Kessler, J., \& Markowitsch, H. J. (2005). Decision-making deficits of Korsakoff patients in a new gambling task with explicit rules: Associations with executive functions. Neuropsychology, 19(3), 267-277. doi:10.1037/0894-4105.19.3.267

Brand, M., Kalbe, E., Kracht, L. W., Riebel, U., Münch, J., Kessler, J., \& Markowitsch, H. J. (2004). Organic and psychogenic factors leading to executive dysfunctions 
in a patient suffering from surgery of a colloid cyst of the Foramen of Monro. Neurocase, 10(6), 420-425. doi:10.1080/13554790490894002

Brand, M., Labudda, K., Kalbe, E., Hilker, R., Emmans, D., Fuchs, G., . . .

Markowitsch, H. J. (2004). Decision-making impairments in patients with

Parkinson's disease. Behavioural neurology, 15(3-4), 77-85.

doi:10.1155/2004/578354

Brand, M., Laier, C., Pawlikowski, M., \& Markowitsch, H. J. (2009). Decision making with and without feedback: The role of intelligence, strategies, executive functions, and cognitive styles. Journal of Clinical and Experimental Neuropsychology, 31(8), 984-998. doi:10.1080/13803390902776860

Brand, M., \& Schiebener, J. (2013). Interactions of age and cognitive functions in predicting decision making under risky conditions over the life span. Journal of Clinical and Experimental Neuropsychology, 35(1), 9-23. doi:10.1080/13803395.2012.740000

Brand, M., Schiebener, J., Pertl, M.-T., \& Delazer, M. (2014). Know the risk, take the win: How executive functions and probability processing influence advantageous decision making under risk conditions. Journal of Clinical and Experimental Neuropsychology, 36(9), 914-929. doi:10.1080/13803395.2014.955783

Burgio, F., Delazer, M., Meneghello, F., Pertl, M.-T., Semenza, C., \& Zamarian, L. (2018). Cognitive training improves ratio processing and decision making in patients with mild cognitive impairment. Journal of Alzheimer's Disease, 64(4), 1213-1226. doi:10.3233/JAD-180461

Cohen, J. (1988). Statistical power analysis for the behavioral sciences (2nd ed.). New York: Academic Press.

de Siqueira, A. S. S., Yokomizo, J. E., Jacob-Filho, W., Yassuda, M. S., \& Aprahamian, I. (2017). Review of decision-making in game tasks in elderly participants with Alzheimer disease and mild cognitive impairment. Dementia and geriatric cognitive disorders, 43, 81-88. doi:10.1159/000455120

Delazer, M., Sinz, H., Zamarian, L., \& Benke, T. (2007). Decision-making with explicit and stable rules in mild Alzheimer's disease. Neuropsychologia, 45(8), 16321641. doi:10.1073/pnas.1108561108

Engelmann, J. B., Capra, C. M., Noussair, C., \& Berns, G. S. (2009). Expert financial advice neurobiologically "offloads" financial decision-making under risk. PloS one, 4(3), e4957. doi:10.1371/journal.pone.0004957

Euteneuer, F., Schaefer, F., Stuermer, R., Boucsein, W., Timmermann, L., Barbe, M. T., ... Kalbe, E. (2009). Dissociation of decision-making under ambiguity and decision-making under risk in patients with Parkinson's disease: a neuropsychological and psychophysiological study. Neuropsychologia, 47(13), 2882-2890. doi:10.1016/j.neuropsychologia.2009.06.014

Figner, B., Mackinlay, R. J., Wilkening, F., \& Weber, E. U. (2009). Affective and deliberative processes in risky choice: Age differences in risk taking in the Columbia Card Task. Journal of Experimental Psychology: Learning, Memory, and Cognition, 35(3), 709-730. doi:10.1037/a0014983

Finucane, M. L., \& Lees, N. B. (2005). Decision-making competence of older adults: Models and methods. Paper presented at the National research Councul Workshop

on Decision Making by Older Adults, Washington DC. 
Finucane, M. L., Mertz, C., Slovic, P., \& Schmidt, E. S. (2005). Task complexity and older adults' decision-making competence. Psychology and aging, 20(1), 71-84. doi:10.1037/0882-7974.20.1.71

Folstein, M. F., Folstein, S. E., \& McHugh, P. R. (1975). "Mini-mental state": a practical method for grading the cognitive state of patients for the clinician. Journal of psychiatric research, 12(3), 189-198.

Freitas, S., Simões, M. R., Alves, L., \& Santana, I. (2013). Montreal cognitive assessment: validation study for mild cognitive impairment and Alzheimer disease. Alzheimer Disease \& Associated Disorders, 27(1), 37-43. doi:10.1097/WAD.0b013e3182420bfe

Gleichgerrcht, E., Ibáñez, A., Roca, M., Torralva, T., \& Manes, F. (2010). Decisionmaking cognition in neurodegenerative diseases. Nature Reviews Neurology, 6(11), 611-623. doi:10.1038/nrneurol.2010.148

Goldberg, T. E., Harvey, P. D., Wesnes, K. A., Snyder, P. J., \& Schneider, L. S. (2015). Practice effects due to serial cognitive assessment: implications for preclinical Alzheimer's disease randomized controlled trials. Alzheimer's \& Dementia: Diagnosis, Assessment \& Disease Monitoring, 1(1), 103-111. doi:10.1016/j.dadm.2014.11.003

Grady, C. L., Haxby, J., Horwitz, B., Sundaram, M., Berg, G., Schapiro, M., . . . Rapoport, S. (1988). Longitudinal study of the early neuropsychological and cerebral metabolic changes in dementia of the Alzheimer type. Journal of Clinical and Experimental Neuropsychology, 10(5), 576-596. doi:10.1080/01688638808402796

Hachinski, V. C., Iliff, L. D., Zilhka, E., Du Boulay, G. H., McAllister, V. L., Marshall, J., . . Symon, L. (1975). Cerebral blood flow in dementia. Archives of neurology, 32(9), 632-637. doi:10.1001/archneur.1975.00490510088009

Hodges, J. R., \& Patterson, K. (1995). Is semantic memory consistently impaired early in the course of Alzheimer's disease? Neuroanatomical and diagnostic implications. Neuropsychologia, 33(4), 441-459. doi:10.1016/00283932(94)00127-B

Hodges, J. R., Salmon, D. P., \& Butters, N. (1990). Differential impairment of semantic and episodic memory in Alzheimer's and Huntington's diseases: a controlled prospective study. Journal of Neurology, Neurosurgery \& Psychiatry, 53(12), 1089-1095. doi:10.1136/jnnp.53.12.1089

Ivnik, R. J., Malec, J. F., Smith, G. E., Tangalos, E. G., \& Petersen, R. C. (1996). Neuropsychological tests' norms above age 55: COWAT, BNT, MAE token, WRAT-R reading, AMNART, STROOP, TMT, and JLO. The Clinical Neuropsychologist, 10(3), 262-278. doi:10.1080/13854049608406689

Lafleche, G., \& Albert, M. S. (1995). Executive function deficits in mild Alzheimer's disease. Neuropsychology, 9(3), 313-320. doi:10.1037/0894-4105.9.3.313

Lejarraga, T., \& Gonzalez, C. (2011). Effects of feedback and complexity on repeated decisions from description. Organizational behavior and human decision processes, 116(2), 286-295. doi:10.1016/j.obhdp.2011.05.001

Liebherr, M., Schiebener, J., Averbeck, H., \& Brand, M. (2017). Decision Making under Ambiguity and Objective Risk in Higher Age-A Review on Cognitive and Emotional Contributions. Frontiers in psychology, 8, 2128. doi:10.3389/fpsyg.2017.02128

MacLeod, C. M., \& Dunbar, K. (1988). Training and Stroop-like interference: Evidence for a continuum of automaticity. Journal of Experimental Psychology: Learning, Memory, and Cognition, 14(1), 126-135. doi:10.1037/0278-7393.14.1.126 
McKhann, G., Drachman, D., Folstein, M., Katzman, R., Price, D., \& Stadlan, E. M. (1984). Clinical diagnosis of Alzheimer's disease: Report of the NINCDSADRDA Work Group under the auspices of Department of Health and Human Services Task Force on Alzheimer's Disease. Neurology, 34(7), 939-939. doi:10.1212/WNL.34.7.939

Morris, R. G., \& Kopelman, M. D. (1986). The memory deficits in Alzheimer-type dementia: A review. The Quarterly Journal of Experimental Psychology Section A, 38(4), 575-602. doi:10.1080/14640748608401615

Morris, S. B., \& DeShon, R. P. (2002). Combining effect size estimates in meta-analysis with repeated measures and independent-groups designs. Psychological methods, 7(1), 105-125.

Mueller, S. M., Schiebener, J., Stöckigt, G., \& Brand, M. (2017). Short-and long-term consequences in decision-making under risk: immediate feedback about longterm prospects benefits people tending to impulsive processing. Journal of Cognitive Psychology, 29(2), 217-239. doi:10.1080/20445911.2016.1245660

Nasreddine, Z. S., Phillips, N. A., Bédirian, V., Charbonneau, S., Whitehead, V., Collin, I., . . Chertkow, H. (2005). The Montreal Cognitive Assessment, MoCA: a brief screening tool for mild cognitive impairment. Journal of the American Geriatrics Society, 53(4), 695-699.

Nelson, H. E. (1976). A modified card sorting test sensitive to frontal lobe defects. Cortex, 12(4), 313-324. doi:10.1016/S0010-9452(76)80035-4

Nelson, P. T., Alafuzoff, I., Bigio, E. H., Bouras, C., Braak, H., Cairns, N. J., . . . Tredici, K. D. (2012). Correlation of Alzheimer disease neuropathologic changes with cognitive status: a review of the literature. Journal of Neuropathology \& Experimental Neurology, 71(5), 362-381. doi:10.1097/NEN.0b013e31825018f7

Perry, R. J., Watson, P., \& Hodges, J. R. (2000). The nature and staging of attention dysfunction in early (minimal and mild) Alzheimer's disease: relationship to episodic and semantic memory impairment. Neuropsychologia, 38(3), 252-271. doi:10.1016/S0028-3932(99)00079-2

Pertl, M.-T., Zamarian, L., \& Delazer, M. (2017). Reasoning and mathematical skills contribute to normatively superior decision making under risk: Evidence from the game of dice task. Cognitive processing. doi:10.1007/s10339-017-0813-x

Schiebener, J., \& Brand, M. (2015a). Decision making under objective risk conditions a review of cognitive and emotional correlates, strategies, feedback processing, and external influences. Neuropsychology review, 25(2), 1-28. doi:10.1007/s11065-015-9285-X

Schiebener, J., \& Brand, M. (2015b). Self-reported strategies in decisions under risk: Role of feedback, reasoning abilities, executive functions, short-term-memory, and working memory. Cognitive processing, 16(4), 401-416. doi:10.1007/s10339-015-0665-1

Schiebener, J., \& Brand, M. (2017). Age-related variance in decisions under ambiguity is explained by changes in reasoning, executive functions, and decision-making under risk. Cognition and emotion, 31(4), 816-824. doi:10.1080/02699931.2016.1159944

Schiebener, J., Wegmann, E., Gathmann, B., Laier, C., Pawlikowski, M., \& Brand, M. (2014). Among three different executive functions, general executive control ability is a key predictor of decision making under objective risk. Frontiers in psychology, 5(1386), 1-13. doi:10.3389/fpsyg.2014.01386 
Schiebener, J., Wegmann, E., Pawlikowski, M., \& Brand, M. (2012). Anchor effects in decision making can be reduced by the interaction between goal monitoring and the level of the decision maker's executive functions. Cognitive processing, 13(4), 321-332.

Schiebener, J., Wegmann, E., Pawlikowski, M., \& Brand, M. (2013). Supporting decisions under risk: explicit advice differentially affects people according to their working memory performance and executive functioning. Neuroscience of decision making, 1, 9-18.

Schiebener, J., Zamarian, L., Delazer, M., \& Brand, M. (2011). Executive functions, categorization of probabilities, and learning from feedback: What does really matter for decision making under explicit risk conditions? Journal of Clinical and Experimental Neuropsychology, 33(9), 1025-1039. doi:10.1080/13803395.2011.595702

Sinz, H., Zamarian, L., Benke, T., Wenning, G., \& Delazer, M. (2008). Impact of ambiguity and risk on decision making in mild Alzheimer's disease. Neuropsychologia, 46(7), 2043-2055. doi:10.1016/j.neuropsychologia.2008.02.002

Skovlund, E., \& Fenstad, G. U. (2001). Should we always choose a nonparametric test when comparing two apparently nonnormal distributions? Journal of clinical epidemiology, 54(1), 86-92. doi:10.1016/S0895-4356(00)00264-X

Spreen, O., \& Strauss, E. (1998). A compendium of neuropsychological tests: Administration, norms, and commentary. New York: Oxford University Press.

Steiger, J. H. (1980). Tests for comparing elements of a correlation matrix. Psychological bulletin, 87(2), 245.

Stroop, J. R. (1935). Studies of interference in serial verbal reactions. Journal of experimental psychology, 18(6), 643-662. doi:10.1037/h0054651

Todd, P., \& Benbasat, I. (1994). The influence of decision aids on choice strategies: an experimental analysis of the role of cognitive effort. Organizational behavior and human decision processes, 60(1), 36-74. doi:10.1006/obhd.1994.1074

Tombaugh, T. N., \& McIntyre, N. J. (1992). The mini-mental state examination: a comprehensive review. Journal of the American Geriatrics Society, 40(9), 922935. doi:10.1111/j.1532-5415.1992.tb01992.x

Wechsler, D., Coalson, D. L., \& Raiford, S. E. (1997). WAIS-III: Wechsler adult intelligence scale: Psychological Corporation San Antonio, TX.

Welsh, K. A., Butters, N., Hughes, J. P., Mohs, R. C., \& Heyman, A. (1992). Detection and staging of dementia in Alzheimer's disease: Use of the neuropsychological measures developed for the Consortium to Establish a Registry for Alzheimer's Disease. Archives of neurology, 49(5), 448-452. doi:10.1001/archneur.1992.00530290030008

Yaniv, I. (2004). Receiving other people's advice: Influence and benefit. Organizational behavior and human decision processes, 93(1), 1-13. doi:10.1016/j.obhdp.2003.08.002

Zamarian, L., Sinz, H., Bonatti, E., Gamboz, N., \& Delazer, M. (2008). Normal aging affects decisions under ambiguity, but not decisions under risk. Neuropsychology, 22(5), 645-657. doi:10.1037/0894-4105.22.5.645 